\title{
A Discrimination Method Between Transformer Inrush and Fault Current Based on Chromatic Monitoring
}

\author{
ZIYAD S. ALMAJALI \\ Department of Electrical Engineering \\ Faculty of Engineering \\ Mutah University \\ Mutah - Karak, \\ JORDAN
}

\begin{abstract}
For successful transformer differential protection employment, correct discrimination between inrush current and fault current is essential. It is one of the main focuses of research and one of the main challenges for transformer protection. In this paper, a discrimination method based on utilizing chromatic monitoring of the box dimension algorithm outcome curve for transformer differential current in time-domain analysis is proposed.

The $\mathrm{x}-\mathrm{L}$ chromatic mapping is employed for general detection of fault cases, while the $\mathrm{x}-\mathrm{y}$ chromatic mapping result is used for distinguishing inrush current from the fault current cycles.

The preliminary results show that the proposed method can effectively provide correct discrimination of the current type within quite a short time and thus help in providing efficient decision-making supportive protection tool.
\end{abstract}

Key-Words: - Transformer, Inrush, Fault, Current, time-domain analysis, Relay, Condition monitoring.

Received: May 10, 2021. Revised: November 25, 2021. Accepted: December 8, 2021. Published: December 23, 2021.

\section{Introduction}

According to power system protection philosophy, all system elements are important, and none may be left without any protection. However, the details and depth of the protection level depend on the importance, cost, role, and location of the protected element. Transformer protection is considered one of the main priorities of system protection, regardless of its location, due to the direful effect of losing it. [1]

The transformer has an initial cost. It can be assumed that its use compensates for this cost with the benefits covered by its function of reducing losses. However, the sudden and unplanned loss of this service component has a negative impact that cannot be ignored. The reliability and quality of the service are interrupted because of the need to completely remove the transformer from service during maintenance in addition to the long time required for repair.

There are many adopted methods for transformer protection. Some of these approaches depend on the effect of fault on the transformer oil, such as Buchholz relays, or by analysis of the oil's dissolved gases, with the aim of early alert to possibly serious faults. However, the main protection schemes employ the current in the form of a fuse or overcurrent relay for protection from high currents. The most commonly adopted and most effective method to discriminate internal from external fault is the current differential method.[2] Employing it creates various challenges; namely, the main challenge is the lack of ability to distinguish between fault current and magnetization inrush current.[3]

Both fault current and magnetization inrush current have a common high magnitude. Differential relays sense a difference in both cases, in different fault conditions as is also the case due to inrush current. This may result in a decision to disconnect parts that do not need to be disconnected and ignoring it may lead to the loss of the transformer. It may even result in successive failures that threaten the entire system.

Accordingly, it is necessary to build or design a tool for accurately distinguishing between the two currents. The relay operation or blocking is linked to decisions issued by such a tool. The most widely adopted methods for addressing such problems are based on utilization of harmonics. The harmonics used in these approaches are the second and, sometimes, fifth harmonics[4]. But the installation 
of a power transmission line over a long distance cause false operation due to the similarity in value of second harmonic in both cases, in the fault current and in the inrush current as well[5].

In addition, relying on the availability of second harmonics in the inrush currents as an undoubted fact may be considered a false strategy because the major sources of harmonics in the monitored inrush currents are the nonlinearities of the transformer's core. However, recent improvements in the design of transformer core materials cause magnitude reduction of these existing harmonics, which leads to dysfunction of the designed tools.[6] or as in the case of ultrasaturation, which can happen when energizing a loaded transformer[7].

Given the importance of the subject, the ambiguity in the distinction between the two currents has attracted the attention of researchers. Neural network solutions [8] and fuzzy logic-based methods[9], in addition to a combination of several artificially based methods, have already been reported.[10]

Most of these artificially based approaches for solving this problem depend on feature extractions' pre-processing for accurate classification. Furthermore, wavelet transform was used and reported in many publications and research works due to its powerful processing. It was also used extensively in many other research fields for its aforementioned characteristics.[11][12] However, these techniques may require a significant computational burden for training.

This paper proposes a discrimination method between inrush current and fault current based on utilizing chromatic monitoring of transformer readings produced by the box dimension algorithms known as grid method, counting box method, or both. First, the box dimension algorithm and its resulting curve concepts are introduced. Then, the curve monitoring is described, as performed in the time domain by the chromatic xyz method. The results mapping allows accurate discrimination of regions, which supports and facilitates an accurate tripping or blocking decision of differential current relays.

\section{The Box Dimension Method Algorithm}

The implementation of the box dimension algorithm (also called the grid method)[13] can be summarized as "full coverage of a monitored signal by a series of small boxes with defined width (d) within in a window of duration (T)." Figure 1 shows an illustration of a sample signal and its squares coverage. The outcome of the counting box method is a number $(\mathrm{N})$, which is evaluated by counting the required boxes (shaded grey in Figure 1) for total coverage. Boxes of a smaller width result in accurate coverage but require higher processing.

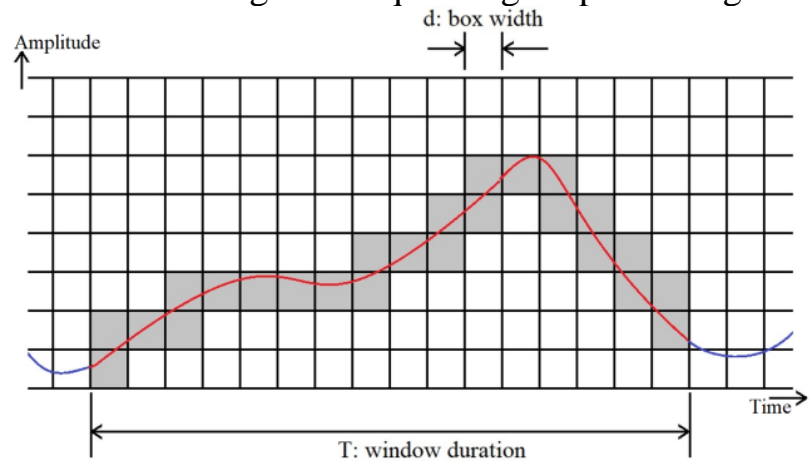

Fig. 1: Box dimension algorithm application

The described method belongs to fractal geometry - the box dimension algorithm is widely adopted in monitoring the complex irregularities of signals[14]; thus, numerous employments with regard to the detection of the start and the end of disturbances were applied successfully in power system monitoring of power quality disturbances. [15]

The procedure for counting the boxes was repeated for a specified sliding length say half wave. The resulting record of the counted boxes with time is called grille curve. The idea originally revolved around searching for what distinguishes the two cases under observation based on the ability of the adopted method in singularities points discovery as mentioned above.

In the case of the inrush current (Figure 2), singularities are available in its non-sinusoidal waveform due to alternating transitions between entering and leaving core saturation discontinuities. which lead to the signal's severe distortion, and this produce a grille curve with a changing value, such as the one shown in Figure 3. In the case of the fault, although it is higher in magnitude, it has an approximate sine wave because of the very low saturation component, which can be ignored. thus produces a constant value grille curve like the one shown in Figure 4. [16]

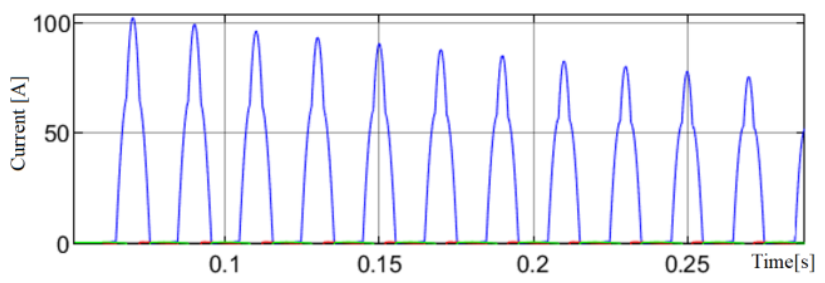

Fig. 2: Inrush current 


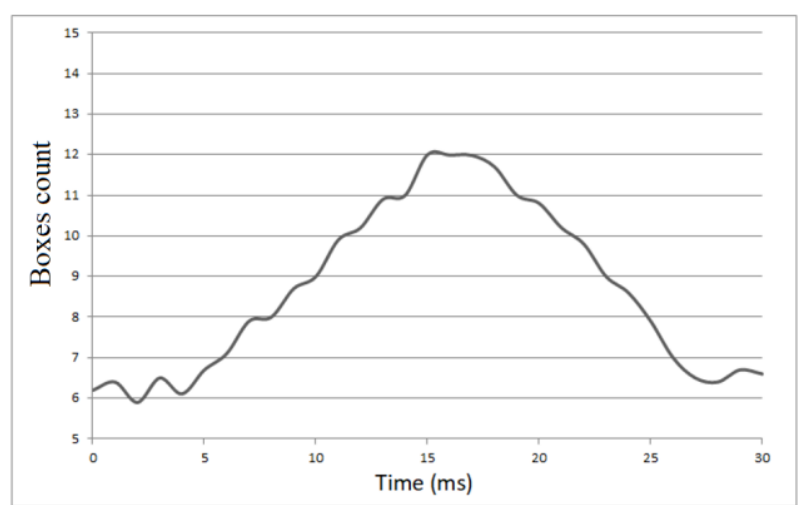

Fig. 3: Count of boxes for inrush current

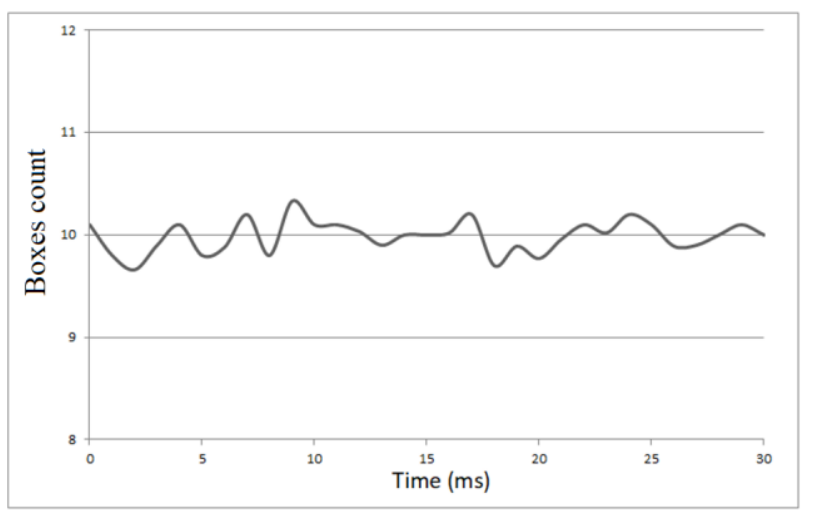

Fig. 4: Count of boxes for Fault current

Although the box dimension algorithm method is considered part of the preprocessing stage in this work, the whole process outcome is highly affected by the outcome of this step since a clear difference is observed in the preliminary results of the two monitored signals (i.e., the fault current and the magnetization inrush current). The classification task is left for the chromatic monitoring stage, which depends on its distinguishing mapping ability, which is introduced in the next section.

\section{Chromatic Monitoring}

Based on the eye's main function of distinguishing colors, chromaticity principles have been adopted in many advanced monitoring applications. The fields of application were not limited only to optics; various fields were included, and more than satisfactory results were achieved in most cases.[17]

The human eye transmits scene details to the brain, where analysis is performed. Using the analyzed scene's information, the brain captures complex variables and analyses them based on acquired training. In this way, it deals with all possibilities of various complex events. The work of the eye reduces a lot of effort required by the brain.
The optical cells in the cornea of the eye are used to distinguish different colours by issuing different reactions and responses according to the magnitude to which the signal covers the visible frequency spectrum. The chromatic idea is based on the principle of the optical cell, but researchers could change the monitored signal type or source, such as choosing transformer current to be monitored, as in this work. Additionally, there is the freedom to change the signal domain to time instead of frequency, for example.

To illustrate, Figure 5 shows three signal processors ( $\mathrm{R}, \mathrm{G}$, and $\mathrm{B}$ ) with slight similarities to those processors found in the human eye. The three processors cover the entire range of a monitored signal domain. The responses of the overlapping three processors depend on the parts of the signals that the individual processor covers.

The three signal processors outcomes are given by the following:

$$
\begin{aligned}
& \mathrm{R}=\int \mathrm{R}(\phi) \mathrm{P}(\phi) \mathrm{d} \phi \\
& \mathrm{G}=\int \mathrm{G}(\phi) \mathrm{P}(\phi) \mathrm{d} \phi \\
& \mathrm{B}=\int \mathrm{B}(\phi) \mathrm{P}(\phi) \mathrm{d} \phi
\end{aligned}
$$

$\mathrm{P}(\phi)$ is the monitored signal, and $\mathrm{R}(\phi), \mathrm{G}(\phi)$, and $\mathrm{B}(\phi)$ are the processors' profiles, where $\phi$ is the monitored signals domain. For simplicity, a triangular processor form has been chosen for the three processors' responses.

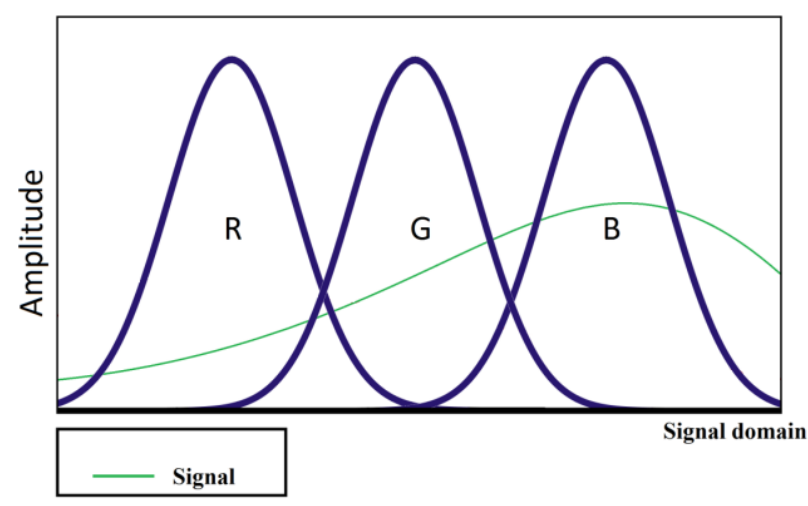

Fig. 5: Chromatic monitoring application

The outputs from the three processors' - R, G, and $\mathrm{B}$ - transformation can be accomplished through various available transformation algorithm options. Due to its simple algorithm, the selected transformation for this work is the xyz transformation, as shown in equations $4-7$, where relative magnitudes $(\mathrm{x}, \mathrm{y}, \mathrm{z})$ for each of the three overlapping processor outputs $(\mathrm{R}, \mathrm{G}, \mathrm{B})$ in addition to signal strength index (L) can be evaluated: 


$$
\begin{aligned}
& x=R / L \\
& y=G / L \\
& z=B / L \\
& L=(R+G+B)
\end{aligned}
$$

Feature extraction through signal distinguishing can be arranged following the chromatic transformation processing stage in a further stage of mapping, which is included in Section 5. The mapping may be arranged by graphical representation for only two of the chromatic parameters shown in the above-mentioned equations since any two of them contain partial but sufficient information about the original signal.

\section{Simulated System}

Simulations for different cases were performed on the system, as shown in Figure 6. A generator rated $225 \mathrm{kVA}$ supplies its load through a 1 MVA, 2400 $\mathrm{V} / 600 \mathrm{~V}, \quad 60 \mathrm{~Hz}$ transformer. The different simulations involve parameter variation of normal inrush current cases in addition to simulation of various faulty conditions are applied using SimPower Toolbox in MATLAB and Simulink.

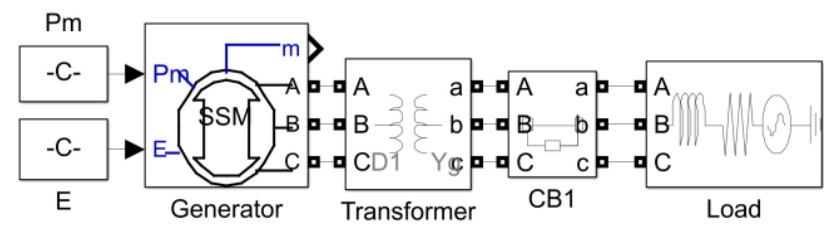

Fig. 6: Simulated experimental system

The purpose of carrying out the experiment and collecting data of different cases is to verify the effectiveness of the proposed methods and their robustness under different operating conditions.

The procedures used in the simulation are summarized in the flowchart portions of Figure 7. The flowchart includes five main steps. The first step; Simply by collecting transformer differential currents under all test cases followed by the second step which is the preprocessing stage of the box dimension algorithm application described in section 2. The chromatic processing starts by flowchart steps three and four where the resulting curve of the box dimension algorithm is fed to chromatic RGB signal filters and the resulting RGB outcome are then transformed by chromatic $\mathrm{x}, \mathrm{y}, \mathrm{z}$ processing stage, steps three and four are introduced in section 3. The classification of the transformer currents is based on the chromatic parameters mapping which is shown as the fifth step in the flowchart.

\section{Results and Discussion}

The procedure described in Figure 7 is applied to transformer differential current under different switching for the testing of inrush currents, as well as different faults: single phase to ground, phase to phase, phase to phase to ground, and three-phase fault currents.

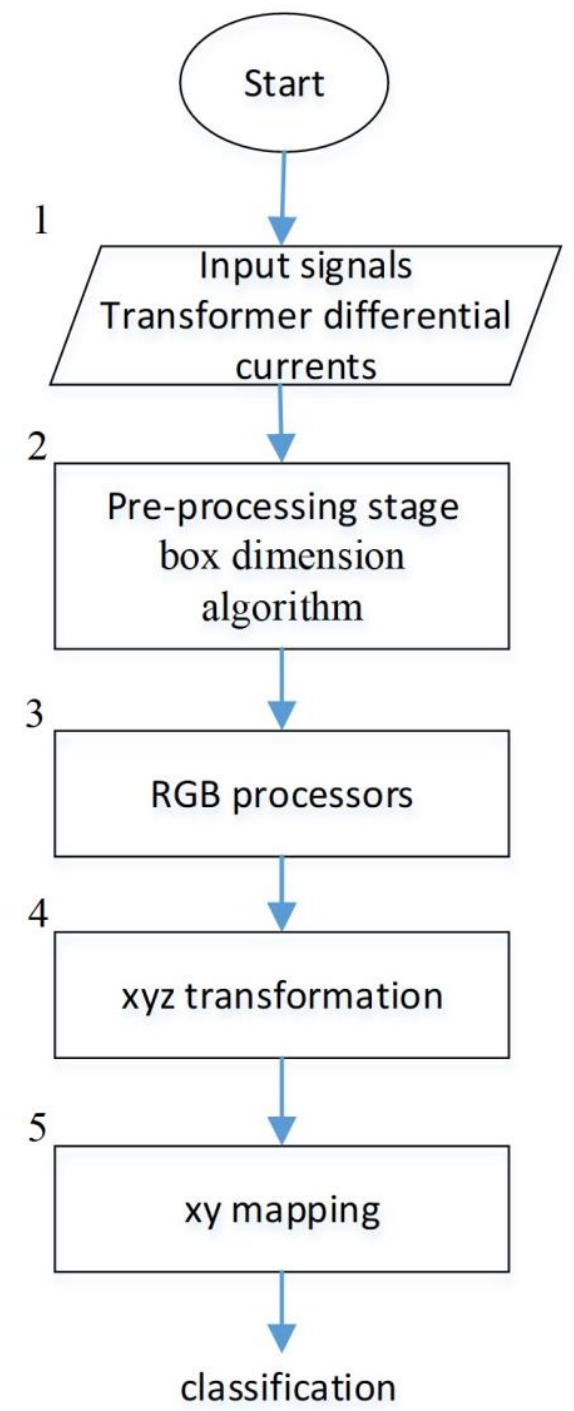

Fig. 7: Method flowchart

Regarding the interrelationship between the chromatic monitoring transformation parameters, the relationship between two variables, namely $\mathrm{x}$ and $y$, is shown in Figure 8, which identifies a significant defining characteristic.

Repeating the test cases with different conditions for sensitivity investigation leads to the formation of clusters, which are clearly distinguishable. A sample of these results is shown on the chromatic $\mathrm{x}-\mathrm{y}$ mapping diagram in Figure 8. The figure shows how the inrush current cluster is distinguishable from the all faults cluster. 
Figure 8 shows the chromatic diagrams y:x, which include various results from the present tests. Although the results are promising, it is worth noting that these are the results of a few starting initial cycles. As the time progressed for the inrush case, it was noted that it took a trace to the original location of the fault cluster, as illustrated in Figure 9, which illustrates the whole monitoring process.

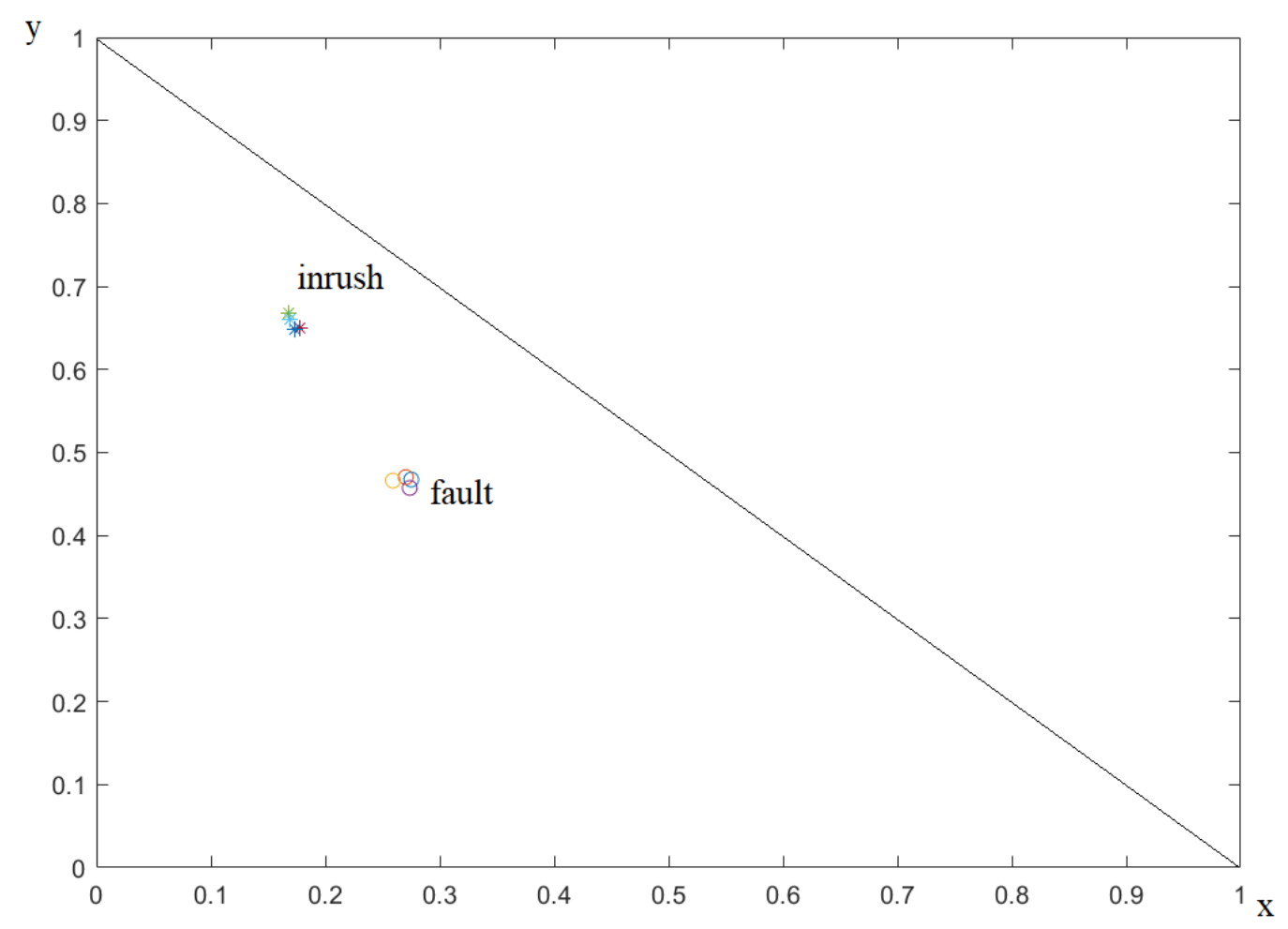

Fig. 8: x-y mapping diagram

A 15-second duration of inrush current simulation is shown in Figure 9. Four short periods are then selected from the simulation period and labelled from 1 to 4 . The proposed algorithm stages are applied to the four selected periods, in addition to one faulty case, for comparison.

With the time progress from 1 to 4 , an increase was recorded in the $\mathrm{x}$ parameter value, while a reduction was recorded in the y parameter change. The location traced its way as time progressed to a coordinate almost similar to the recorded coordinate of the faulty cycles.

The similarity is due to the vanishing of the saturation component of the signal with time progression, and a sinewave is the shape of both cases.

Figure 10 shows mapping locations for both faulty cycles and healthy cycles in addition to inrush current cycles after long enough time passing. By zooming into the cluster, the ambiguity between both cases is illustrated.

The results shown on the chromatic diagrams in Figure 10 lead to the conclusion that faulty cycles in the proposed solution are not distinguishable from the healthy cycles or inrush current cycles after a long enough period of time passes. The objective of the work was to distinguish inrush from fault at the initial operation of the transformer, which is achieved by mapping the initial cycles, as shown in Figure 8. However, another mapping from the same transformation method, but between another pair of parameters, can serve the purpose of distinguishing between fault and healthy, which is the L-x plot. A sample of such results is presented in Figure 11.

Results from both mapping diagrams are to be combined to aid the relay operation by successful distinguishing ability. 


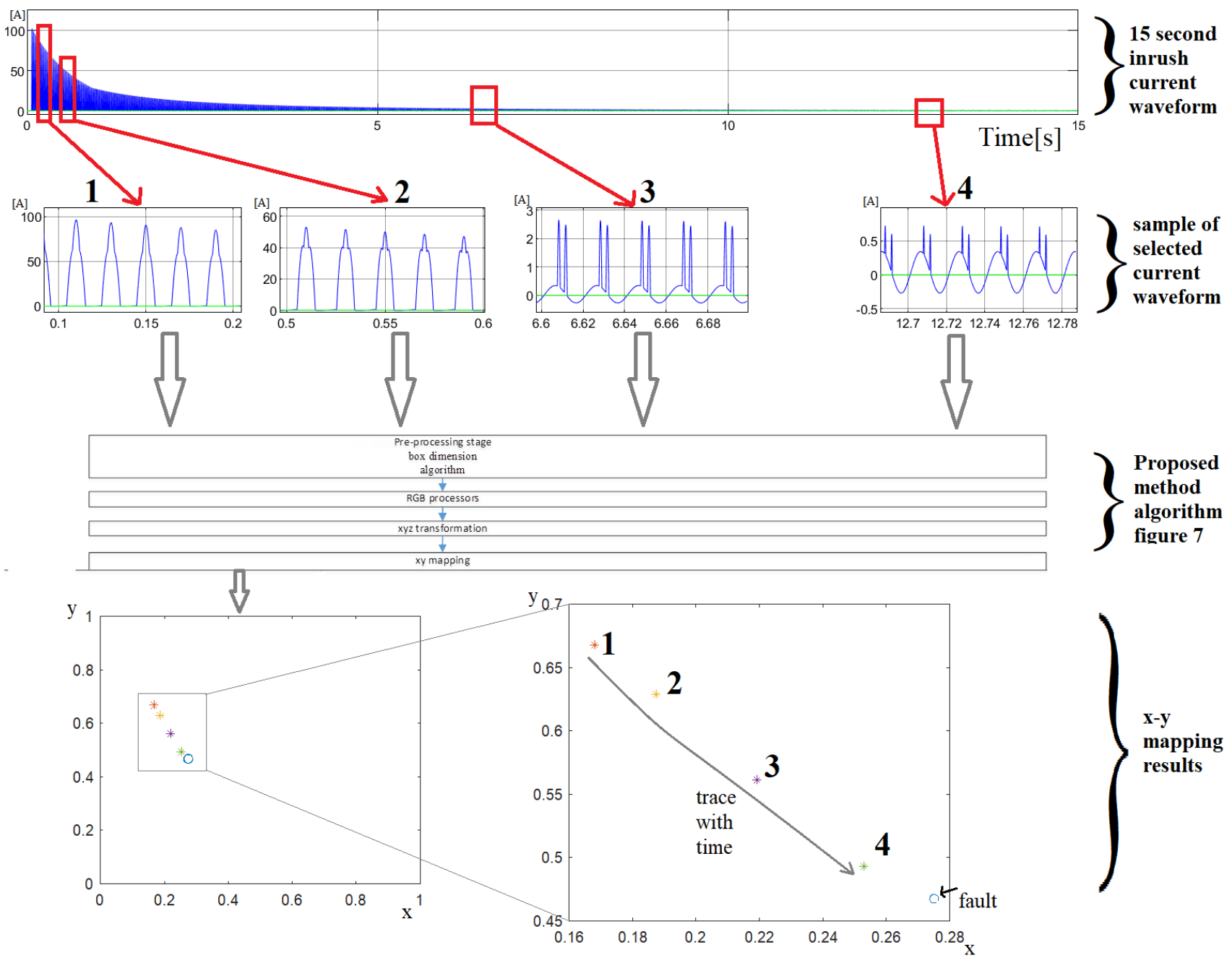

Fig. 9: Trace of the $x-y$ mapping diagram for the inrush case with time progress

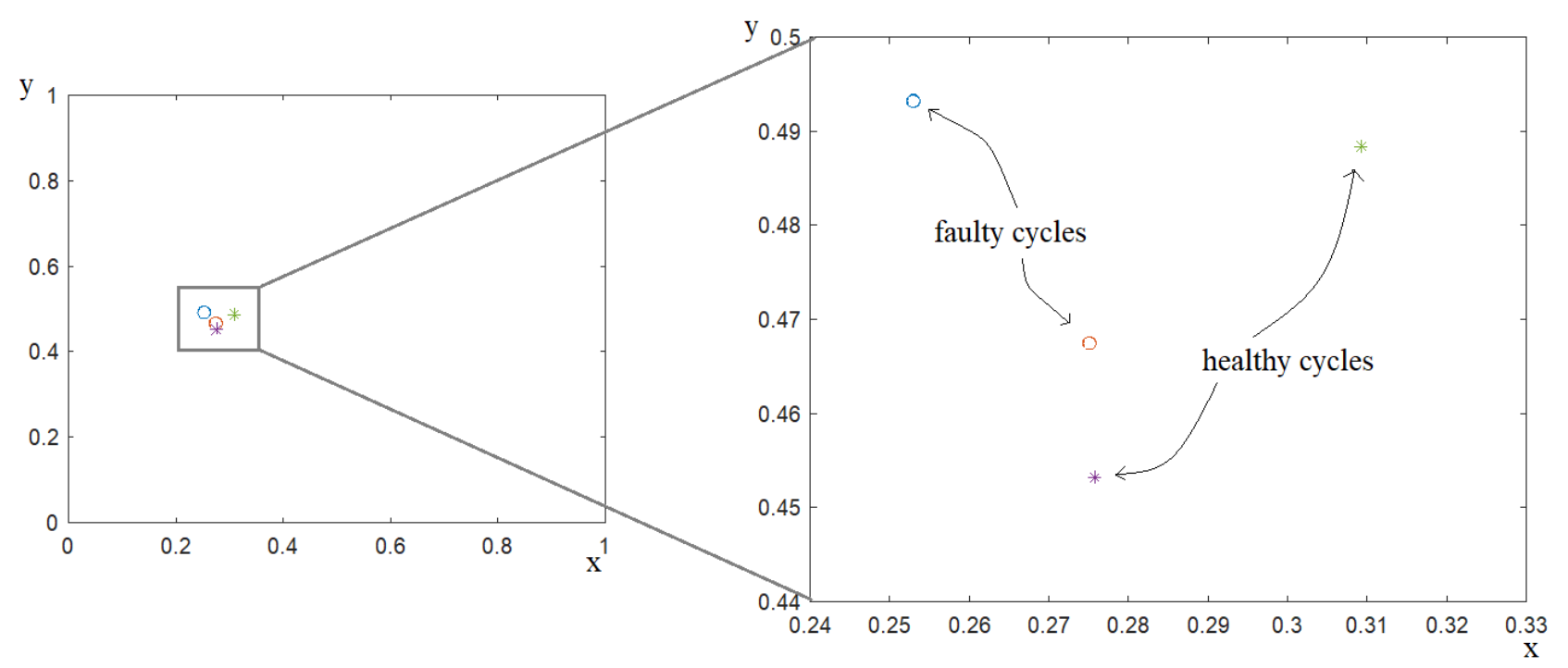

Fig. 10: $x-y$ mapping diagram for both fault and healthy cases 


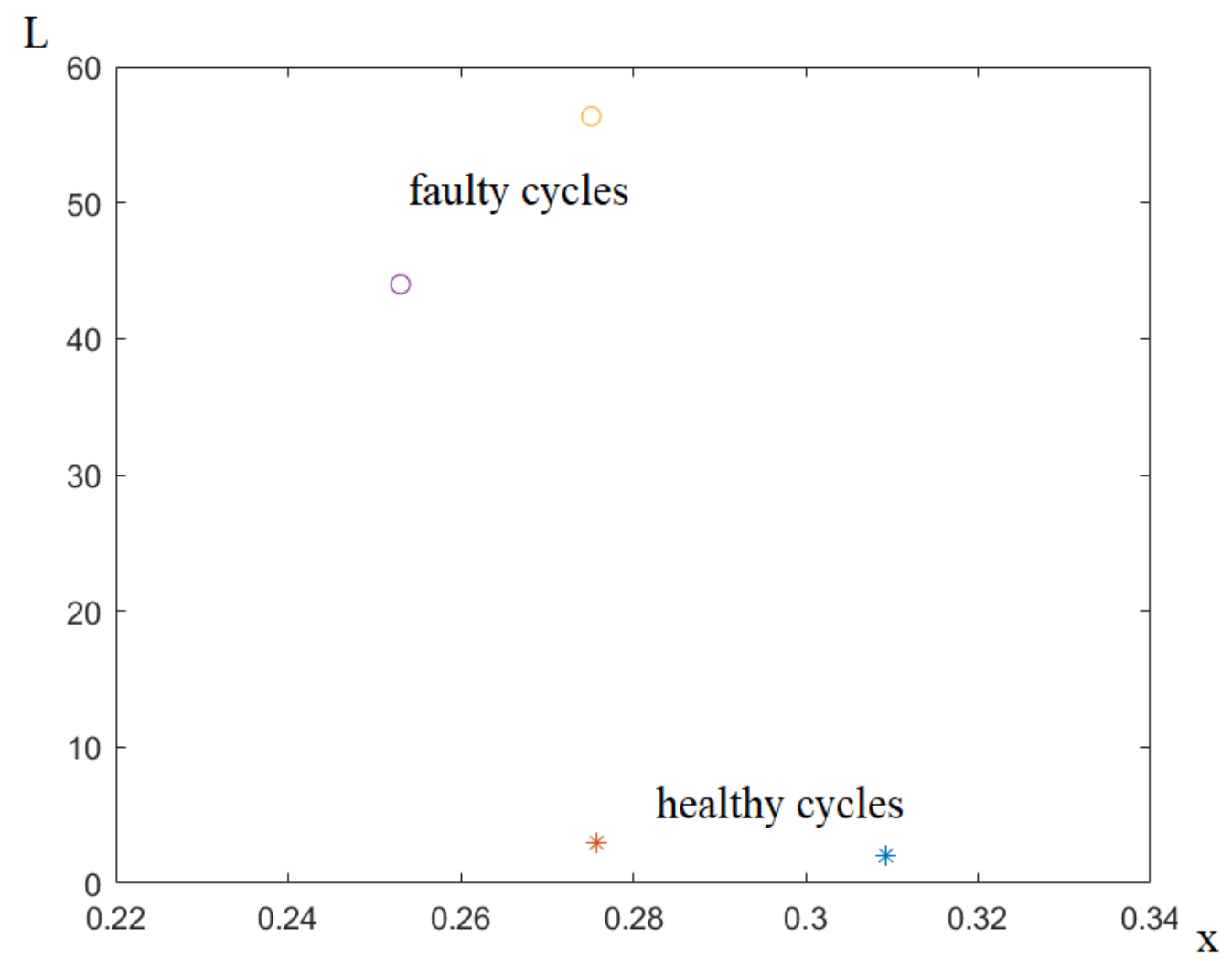

Fig. 11: L-x mapping diagram for both fault and healthy cases

\section{Conclusion}

Preliminary results obtained with chromatic monitoring of box dimension algorithm-generated curve have been presented. An xyz chromatic analysis of the counted boxes has the potential to distinguish between transformer inrush current and fault current. A limitation in the classification process was encountered with time progress of the monitored current waveform and this was solved by another mapping from the same xyz transformation, the L-x diagram shows the potential of distinguishing between the fault and healthy cycles regardless of the time.

Further development of the approach is inspired from the flexibility of chromatic characterization, which offers the possibility of addressing complexities in different domains. Suggestions for future includes work may occur through the usage of different chromatic transformation may be left for future research and investigations with objective of detailed diagnosis through a tool that distinguishes different faults from each other And/or deploying the chromatic analysis for earlier detection of potential problems before causing major losses.

According to the small duration of the required monitored slide window and the given high discrimination of accuracy and response, the proposed method can be classified as providing a reliable, selective, and sensitive protection tool.

\section{References:}

[1] "IEEE Guide for Protecting Power Transformers," in IEEE Std C37.91-2021 (Revision of IEEE Std C37.91-2008), vol., no., pp.1-160, 29 June 2021, doi: 10.1109/IEEESTD.2021.9471045.

[2] K. Antunes Tavares and K. Melo Silva, "Evaluation of Power Transformer Differential Protection Using the ATP Software, " in IEEE Latin America Transactions, vol. 12, no. 2, pp. 161-168, March 2014, doi: 10.1109/TLA.2014.6749533.

[3] H. J. Ferrer, E. O. Schweitzer III "Modern Solutions for Protection, Control and Monitoring of Electric Power Systems" 2010 Quality Books, Inc. ISBN : 9780972502634

[4] K. Behrendt, N. Fischer, and C. Labuschagne "Considerations for Using Harmonic Blocking and Harmonic Restraint Techniques on Transformer Differential Relays" Schweitzer Engineering Laboratories, Inc. SEL Journal of Reliable Power, Volume 2, Number 3, September 2011 
[5] P. Liu, O. P. Malik, C. Chen, G. S. Hope, and Y. Guo, "Improved operation of differential protection of power transformers for internal faults," IEEE Trans. Power Del., vol. 7, no. 4, pp. 1912-1919, Oct. 1992.

[6] S. Urooj, T. Singh, M. Amir and M. Tariq, "Optimal Design of Power Transformer with Advance Core Material using ANSYS Technique" EJECE, European Journal of Electrical Engineering and Computer Science, Vol. 4, No. 5, October 2020

[7] S. Hodder, B. Kasztenny, N. Fischer and Y. $\mathrm{Xia}$, "Low second-harmonic content in transformer inrush currents - Analysis and practical solutions for protection security," 2014 67th Annual Conference for Protective Relay Engineers, 2014, pp. 705-722, doi: 10.1109/CPRE.2014.6799037.

[8] L. G. Perez, A. J. Flechsig, J. L. Meador and Z. Obradovic, "Training an artificial neural network to discriminate between magnetizing inrush and internal faults," in IEEE Transactions on Power Delivery, vol. 9, no. 1, pp. 434-441, Jan. 1994, doi: 10.1109/61.277715.

[9] M.-C. Shin, C.-W. Park and J.-H. Kim, "Fuzzy logic-based relaying for large power transformer protection," in IEEE Transactions on Power Delivery, vol. 18, no. 3, pp. 718-724, July 2003, doi: 10.1109/TPWRD.2003.813598.

[10] A. Esmaeilian, M. Mohseninezhad, M. Khanabadi and M. Doostizadeh, "A novel technique to identify inrush current based on adaptive neuro fuzzy," 2011 10th International Conference on Environment and Electrical Engineering, 2011, pp. 1-4, doi: 10.1109/EEEIC.2011.5874743.

[11] P. R. Gondane, R. M. Sheikh, K. A. Chawre, V. V. Wasnik, A. Badar and M. T. Hasan, "INRUSH CURRENT DETECTION USING WAVELET TRANSFORM AND ARTIFICIAL NEURAL NETWORK," 2018 Second International Conference on Computing Methodologies and Communication (ICCMC), 2018, pp. 866-868, doi: 10.1109/ICCMC.2018.8487832.

[12] O. A. S. Youssef, "Applications of fuzzylogic-wavelet-based techniques for transformers inrush currents identification and power systems faults classification," IEEE PES Power Systems Conference and Exposition, 2004., 2004, pp. 553-559 vol.1, doi: 10.1109/PSCE.2004.1397423.

[13] D. Tuia, M. Kanevski, "Environmental Monitoring Network Characterization and
Clustering" chapter In: Advanced Mapping of Environmental Data Wiley-ISTE ISBN 9781848210608 - p. 19 - 46. DOI https://doi.org/10.1002/9780470611463.ch2, 2008

[14] Z. Xiaofei and X. Dazhuan, "Singular signal detection with fractal," International Conference on Neural Networks and Signal Processing, 2003. Proceedings of the 2003, 2003, pp. 608-611 Vol.1, doi: 10.1109/ICNNSP.2003.1279347.

[15] R. H. Huang, F. H. Wang, J. Zhang and R. C. Duan, "Detection and location of voltage sags based on mathematical morphology and grille fractal," 2014 IEEE PES T\&D Conference and Exposition, 2014, pp. 1-5, doi: 10.1109/TDC.2014.6863302.

[16] W. C. Wu, M. S. Li, T. Y. Ji and Q. H. Wu, "Fast identification of inrush current using a weighted morphological approach," 2013 IEEE PES Asia-Pacific Power and Energy Engineering Conference (APPEEC), 2013, pp. 1-5, doi: 10.1109/APPEEC.2013.6837265.

[17] G. R. Jones, J.W. Spencer, "Advanced Chromatic Monitoring" 2020, CRC Press, https://doi.org/10.1201/9780367815202, eBook ISBN9780367815202.

\section{Creative Commons Attribution License 4.0 (Attribution 4.0 International, CC BY 4.0)}

This article is published under the terms of the Creative Commons Attribution License 4.0 https://creativecommons.org/licenses/by/4.0/deed.en US 